\title{
IDEMPOTENT STATES ON LOCALLY COMPACT GROUPS AND QUANTUM GROUPS
}

\author{
PEKKA SALMI \\ Dedicated to Professor Victor Shulman on the occasion of his 65th birthday
}

\begin{abstract}
This is a short survey on idempotent states on locally compact groups and locally compact quantum groups. The central topic is the relationship between idempotent states, subgroups and invariant $\mathrm{C}^{*}$-subalgebras. We concentrate on recent results on locally compact quantum groups, but begin with the classical notion of idempotent probability measure. We also consider the 'intermediate' case of idempotent states in the Fourier-Stieltjes algebra: this is the dual case of idempotent probability measures and so an instance of idempotent states on a locally compact quantum group.
\end{abstract}

This is a short survey on idempotent states on locally compact groups and, more generally, on locally compact quantum groups. Idempotent states arise for example as limits of random walks (as we shall see in section 11) and as limits in ergodic theorems for random walks [8. Idempotent states are also connected to the construction of the Haar measure of a compact group: taking the Cesàro limit of convolution powers of a probability measure gives an idempotent probability measure, which is the Haar measure if the original measure is suitably chosen. The same process works in the case of compact quantum groups: this is the construction of Haar state due to Woronowicz [42, 45]. Very recently, idempotent states on locally compact quantum groups have also turned up in connection with Hopf images 3 and Poisson boundaries [16].

As we shall see, idempotent states are inherently related to subgroups. However, there is some evidence against the preceding claim, such as Pal's example of an idempotent state on the KacPaljutkin quantum group that does not arise from the Haar state of a subgroup [24]. In this paper we shall see that in fact also Pal's example is associated with a subgroup (in a different way), and so perhaps there is still hope to connect all idempotent states to subgroups, quotient groups or combinations of these. There are not many new things in this survey article: only a cute new proof to a known result and the already-mentioned insight to the example of Pal. Many of the results, and much more, can be found in the recent papers due to (combinations of) Franz, Skalski, Tomatsu and the author $[9,10,11,31,33]$.

\section{RANDOM WALKS AND IDEMPOTENT PROBABILITY MEASURES}

Every probability measure $\mu$ on a discrete group $G$ determines a random walk: if we start from point $s \in G$, then the probability for taking a step to $t \in G$ is

$$
P(s \mapsto t)=\mu_{t s^{-1}},
$$

where $\mu_{t s^{-1}}:=\mu\left(\left\{t s^{-1}\right\}\right)$. Suppose that we start from the identity $e$ and $X_{k}$ is the random variable denoting the position after $k$ steps. We can use convolution to describe the random walk:

$$
\begin{aligned}
P\left(X_{1}=t\right) & =\mu_{t} \\
P\left(X_{2}=t\right) & =\sum_{s \in G} P\left(X_{1}=s\right) P(s \mapsto t)=\sum_{s \in G} \mu_{s} \mu_{t s^{-1}}=(\mu \star \mu)_{t} \\
& \vdots \\
P\left(X_{k}=t\right) & =\left(\mu^{\star k}\right)_{t} .
\end{aligned}
$$

2010 Mathematics Subject Classification. Primary 60B15; Secondary 43A05, 43A35, 46L30, 81R50. 
In general the convolution of measures $\mu$ and $\nu$ on a locally compact group $G$ is defined by

$$
\langle\mu \star \nu, f\rangle=\iint f(s t) d \mu(s) d \nu(t) \quad\left(f \in \mathrm{C}_{0}(G)\right)
$$

here, and throughout the paper, we consider measures on $G$ as functionals on the $\mathrm{C}^{*}$-algebra $\mathrm{C}_{0}(G)$ of continuous functions on $G$ vanishing at infinity. In the discrete case the convolution boils down to

$$
\mu \star \nu=\sum_{s, t \in G} \mu_{s} \nu_{t} \delta_{s t}=\sum_{s \in G}\left(\sum_{t \in G} \mu_{s t^{-1}} \nu_{t}\right) \delta_{s}
$$

where $\delta_{s}$ denotes the Dirac measure at $s$.

More generally, a probability measure on a locally compact group determines a random walk on that group. From this point of view, we shall see that random walks give rise to idempotent probability measures. An idempotent probabity measure on a locally compact group $G$ is a probability measure $\mu$ on $G$ that is an idempotent under the convolution product:

$$
\mu \star \mu=\mu \text {. }
$$

Now suppose that $G$ is a compact group and $\nu$ is a probability measure on $G$. If the sequence of convolution powers of $\nu$ converges in the weak* topology, then the limit is an idempotent probability measure, which embodies the limit of the random walk. The convergence of such a sequence of convolution powers is widely studied in probability theory (see for example [12, 13]).

Taking a slightly different approach, consider the Cesàro averages

$$
\frac{1}{n} \sum_{k=1}^{n} \nu^{\star k}
$$

The sequence of these averages always converges in the weak* topology. Morevover, the limit $\mu$ is an idempotent probability measure. This gives a useful way to generate idempotent probability measures: for example, the Haar measure of a compact group may be constructed this way. (In the case of non-compact groups the situation is more complicated and to make sure that the limit is non-zero, some form of tightness needs to be assumed for the sequence of convolution powers of $\nu$.

\section{KAWADA-ITÔ THEOREM}

Now that we have seen how idempotent probability measures may arise in practice, a natural question is how to characterise these measures. In the case of locally compact abelian groups, we may use the Fourier-Stieltjes transform to convert an idempotent measure to a characteristic function on the dual group. This trivialises the algebraic side of things, but now the positivity and the normalisation condition become non-trivial. Still, this is a useful approach and, as we shall see in the next section, leads to a simple characterisation. But let us first review the history of the general problem.

Already in 1940 Kawada and Itô [17] characterised idempotent probability measures on compact groups as the normalised Haar measures of compact subgroups. It seems that harmonic analysts were unaware of this paper, and Wendel [41] rediscovered the result in 1954 (truth be told, Wendel's main result is an interesting new proof for the existence of Haar measure on a compact group $G$, using idempotents in the compact semigroup formed by the probability measures on $G$ ). Trying to characterise all idempotent measures on a locally compact abelian group, Rudin [29, 30] showed that any idempotent measure is concentrated on a compact subgroup, thereby extending Wendel's result - or that of Kawada-Itô - to locally compact abelian groups. (The full description of idempotent measures on locally compact abelian groups is due to Cohen [4]; the non-abelian case is open.) Independently, Pym [28] and Loynes 23] characterised idempotent probability measures on locally compact groups (not necessarily abelian) as the normalised Haar measures of compact subgroups. However, since the problem took two separate paths, one starting from Kawada-Itô and another one from Wendel, it is perhaps not that surprising that the problem was solved already in 1954, by Kelley [18. The three approaches, due to Kelley, Pym and Loynes, are all quite different: Kelley studies operators on $\mathrm{C}_{0}(G)$, Pym idempotent measures on semigroups and 
Loynes operator-valued Fourier transform. There are also other generalisations, for example one due to Parthasarathy [25] to complete separable metric groups, which need not be locally compact.

Theorem 1 (Kawada-Itô). Let $\mu$ be an idempotent probability measure on a locally compact group $G$. Then there is a compact subgroup $H$ of $G$ such that $\mu$ is the normalised Haar measure of $H$ (considered as a measure on $G$ ).

The Kawada-Itô theorem gives a procedure to construct the Haar measure of a compact group. Start with a probability measure $\nu$ whose support generates the compact group $G$. As mentioned in the preceding section, the Cesàro averages of convolution powers of $\nu$ converge in the weak* topology to an idempotent probability measure. It follows from the Kawada-Itô theorem, that the limit is the normalised Haar measure of $G$ due to the choice of $\nu$.

As another example, consider the circle group $\mathbb{T}$ and the Dirac measure $\delta_{z}$ at some $z \in \mathbb{T}$. Then there is an idempotent probability measure $\mu$ such that $\frac{1}{n} \sum_{k=1}^{n} \delta_{z}^{* k} \rightarrow \mu$ weak ${ }^{*}$. If $z$ is a rational multiple of $\pi$, then $\mu$ is the counting measure of the finite subgroup of $\mathbb{T}$ generated by $z$. On the other hand, if $z$ is an irrational multiple of $\pi$, then $\mu$ is the normalised Lebesgue measure on $\mathbb{T}$. The latter statement amounts to the Weyl equidistribution theorem.

\section{IDEmpotent States in the Fourier-StieltJes Algebra}

Let $G$ be a locally compact group. The Fourier-Stieltjes algebra $\mathrm{B}(G)$ is the collection of all coefficient functions $(\pi(\cdot) \xi \mid \zeta$ ) of strongly continuous unitary representations $\pi$ of $G$ (here $\xi$ and $\zeta$ are elements of the representation space $\left.\mathcal{H}_{\pi}\right)$. The Fourier-Stieltjes algebra is the dual space of the universal group $\mathrm{C}^{*}$-algebra $\mathrm{C}^{*}(G)$ (this determines the norm of $\mathrm{B}(G)$ ), and $\mathrm{B}(G)$ is a Banach algebra under the pointwise multiplication of functions. If $G$ is abelian, $\mathrm{B}(G)$ is isomorphic, via Fourier-Stieltjes transform, to the measure algebra $\mathrm{M}(\widehat{G})$ of Radon measures on the dual group $\widehat{G}$.

An idempotent state in $\mathrm{B}(G)$ is a state on $\mathrm{C}^{*}(G)$ that is an idempotent:

$$
u^{2}=u \text {. }
$$

That $u$ is a state means that $u$ is a positive definite function with $u(e)=1$, where $e$ denotes the identity of $G$. In the case of abelian $G$, the Fourier-Stieltjes transform takes an idempotent probability measure on $\widehat{G}$ to an idempotent state in $\mathrm{B}(G)$. This explains why the characterisation of idempotent states in $\mathrm{B}(G)$ may be viewed as the dual version of the Kawada-Itô theorem. In fact, many early studies on idempotents in $\mathrm{M}(G)$, with $G$ abelian, used the Fourier-Stieltjes transform to translate the problem to the dual setting.

Continuing from the work of Cohen [4], mentioned in the previous section, Host [14] characterised all idempotents in $\mathrm{B}(G)$ as characteristic functions of sets in the open coset ring of $G$. However, his characterisation does not immediately lead to the following characterisation of idempotent states, which is due to Ilie and Spronk [15. The short proof presented here is new (Ilie and Spronk obtained their result as a corollary of a more general characterisation of contractive idempotents).

Theorem 2. Every idempotent state in $\mathrm{B}(G)$ is the characteristic function of an open subgroup of $G$.

Proof. First of all, every idempotent in $\mathrm{B}(G)$ is a characteristic function of some open (and closed) set $H$, because $\mathrm{B}(G)$ consists of continuous functions. Denote the universal representation of $G$ by $\varpi$. If $s \in H$, then

$$
u\left(s^{-1}\right)=\left\langle u, \varpi\left(s^{-1}\right)\right\rangle=\left\langle u, \varpi(s)^{*}\right\rangle=\overline{\langle u, \varpi(s)\rangle}=1,
$$

so $s^{-1}$ is also in $H$. Moreover,

$$
\left\langle u, \varpi(s)^{*} \varpi(s)\right\rangle=u(e)=1
$$

because $u$ is a state, and

$$
\left\langle u, \varpi(s)^{*}\right\rangle\langle u, \varpi(s)\rangle=1 \cdot 1=1 .
$$


It follows from Choi's theorem on multiplicative domains (see [26. Theorem 3.19]) that $u$ is multiplicative at $\varpi(s)$. So for every $t \in G$,

$$
u(t s)=u(t) u(s)
$$

which implies that $H$ is closed under multiplication. Hence $H$ is an open subgroup and $u=1_{H}$.

The fact that compact subgroups in the Kawada-Itô theorem have changed to open ones in the preceding result reflects subgroup duality. Suppose that $G$ is abelian and $H$ is a closed subgroup of $G$. Then the continuous characters on $G$ that are constant 1 on $H$ form a closed subgroup $H^{\perp}$ of $\widehat{G}$. Now $H$ is compact if and only if $H^{\perp}$ is open, and vice versa. Moreover, the Fourier-Stieltjes transform maps a measure on $G$ supported by $H$ to a function on $\widehat{G}$ that is constant on the cosets of $H^{\perp}$.

\section{LOCALLY COMPACT QUANTUM GROUPS}

Locally compact quantum groups provide a natural context to discuss the results in the previous sections in a unified manner. We shall walk through the definition due to Kustermans and Vaes [21. Let $\mathbb{G}$ denote a locally compact quantum group. This means that we have a $\mathrm{C}^{*}$-algebra $\mathrm{C}_{0}(\mathbb{G})$, a non-degenerate $*$-homomorphism $\Delta: \mathrm{C}_{0}(\mathbb{G}) \rightarrow \mathrm{M}\left(\mathrm{C}_{0}(\mathbb{G}) \otimes \mathrm{C}_{0}(\mathbb{G})\right.$ ) (where the tensor product is the minimal $\mathrm{C}^{*}$-algebraic tensor product and $\mathrm{M}(\cdot)$ denotes the multiplier algebra) such that

$$
(\mathrm{id} \otimes \Delta) \Delta=(\Delta \otimes \mathrm{id}) \Delta \quad(\text { coassociativity })
$$

and

$$
\overline{\operatorname{span}} \Delta\left(\mathrm{C}_{0}(\mathbb{G})\right)\left(\mathrm{C}_{0}(\mathbb{G}) \otimes 1\right)=\overline{\operatorname{span}} \Delta\left(\mathrm{C}_{0}(\mathbb{G})\right)\left(1 \otimes \mathrm{C}_{0}(\mathbb{G})\right)=\mathrm{C}_{0}(\mathbb{G}) \otimes \mathrm{C}_{0}(\mathbb{G}) .
$$

The map $\Delta$ is called the comultiplication of $\mathbb{G}$. We also need to assume that there exist left and right Haar weights on $\mathrm{C}_{0}(\mathbb{G})$, denoted by $\phi$ and $\psi$, respectively. These are so-called KMS-weights, which are densely defined, faithful and lower semicontinuous. The important invariance properties, as with Haar measures, are that

$$
\phi((\omega \otimes \mathrm{id}) \Delta(a))=\omega(1) \phi(a)
$$

and

$$
\psi((\mathrm{id} \otimes \omega) \Delta(b))=\omega(1) \psi(b)
$$

whenever $\omega \in \mathrm{C}_{0}(\mathbb{G})_{+}^{*}$ and $a, b \in \mathrm{C}_{0}(\mathbb{G})_{+}$are such that $\phi(a)<\infty$ and $\psi(b)<\infty$. So a locally compact quantum group is given by a $\mathrm{C}^{*}$-algebra that has a suitable comultiplication and left and right Haar weights. It is convenient to use the suggestive notation $\mathrm{C}_{0}(\mathbb{G})$ for the $\mathrm{C}^{*}$-algebra because in the commutative case the $\mathrm{C}^{*}$-algebra is $\mathrm{C}_{0}(G)$ for some locally compact group $G$. In this case the comultiplication is given by dualised group multiplication:

$$
\Delta(f)(s, t)=f(s t) \quad\left(f \in \mathrm{C}_{0}(G), s, t \in G\right) .
$$

Note that $\Delta(f) \in \mathrm{C}_{b}(G \times G)=\mathrm{M}\left(\mathrm{C}_{0}(G) \otimes \mathrm{C}_{0}(G)\right)$ but $\Delta(f) \notin \mathrm{C}_{0}(G) \otimes \mathrm{C}_{0}(G)$ unless $G$ is compact or $f=0$. Of course the left and right Haar weights are given by integration against the left and right Haar measures, respectively. Whenever $\mathbb{G}$ is a locally compact quantum group such that $\mathrm{C}_{0}(\mathbb{G})$ is commutative, it is of this form.

Next we consider the dual of the commutative case, which is known as the co-commutative case. Let $\lambda$ be the left regular representation of $G$. Then the reduced group $\mathrm{C}^{*}$-algebra $\mathrm{C}_{r}^{*}(G)$ is generated by $\lambda\left(\mathrm{L}^{1}(G)\right)$ in $\mathrm{B}\left(\mathrm{L}^{2}(G)\right)$. (Hopefully the reader is not confused by the two uses of 'B' as both the Fourier-Stieltjes algebra and the algebra of bounded operators; the distinction should be clear from the context.) We define a comultiplication on $\mathrm{C}_{r}^{*}(G)$ by putting

$$
\Delta(\lambda(s))=\lambda(s) \otimes \lambda(s) \quad(s \in G) .
$$

Note that actually $\lambda(s)$ is in $\mathrm{M}\left(\mathrm{C}_{r}^{*}(G)\right)$ but the above does define a unique comultiplication on $\mathrm{C}_{r}^{*}(G)$, because the linear span of $\lambda(G)$ is strictly dense in $\mathrm{M}\left(\mathrm{C}_{r}^{*}(G)\right)$. In this case the left and right Haar weights coincide and are the so-called Plancherel weight. The construction of this weight uses Tomita-Takesaki theory [34, section VII.3] (for discrete $G, \phi(a)=\left(a \delta_{e} \mid \delta_{e}\right)$ is the usual tracial state). 
Both these examples may be considered as Kac algebras 7]. Every Kac algebra determines a locally compact quantum group, so the latter notion is more general. For an example of a locally compact quantum group that is not a Kac algebra, see section 7 , which includes the description of the quantum deformation of $\mathrm{SU}(2)$ defined by Woronowicz. For a more thorough introduction to locally compact quantum groups, see for example the survey by Kustermans and Tuset [19, 20. or the book by Timmermann [36].

From now on we shall concentrate on locally compact quantum groups $\mathbb{G}$ that are coamenable. That means that there is a state $\epsilon$ on $\mathrm{C}_{0}(\mathbb{G})$, called the counit of $\mathbb{G}$, such that

$$
(\mathrm{id} \otimes \epsilon) \Delta(a)=(\epsilon \otimes \mathrm{id}) \Delta(a)=a
$$

for every $a \in \mathrm{C}_{0}(\mathbb{G})$. In the commutative case coamenability is a vacuous condition (every commutative quantum group is coamenable), but a co-commutative quantum group $\mathbb{G}=\widehat{G}$ is coamenable if and only if the locally compact group $G$ is amenable.

\section{Classical Cases as instances of idempotent States on locally Compact Quantum GROUPS}

The notion of idempotent state from the two classical cases - idempotent probability measures on groups and idempotent states in the Fourier-Stieltjes algebra - is easily generalised to the language of locally compact quantum groups. The dual space of the $\mathrm{C}^{*}$-algebra $\mathrm{C}_{0}(\mathbb{G})$ carries a natural Banach algebra structure: the multiplication is defined by

$$
\omega \star \sigma(a)=(\omega \otimes \sigma) \Delta(a) \quad\left(\omega, \sigma \in \mathrm{C}_{0}(\mathbb{G})^{*}, a \in \mathrm{C}_{0}(\mathbb{G})\right) .
$$

An idempotent state on a locally compact quantum group $\mathbb{G}$ is a state $\omega$ on the $\mathrm{C}^{*}$-algebra $\mathrm{C}_{0}(\mathbb{G})$ that is an idempotent under the product defined above: $\omega \star \omega=\omega$.

A much more difficult task than the definition is to unify the results from the classical cases to general results on locally compact quantum groups. Indeed, it is perhaps not even possible to do so. To even bring forth this discussion we need some further terminology.

A locally compact quantum group $\mathbb{H}$ is compact if $\mathrm{C}_{0}(\mathbb{H})$ is unital, in which case we write $\mathrm{C}(\mathbb{H})$ for $\mathrm{C}_{0}(\mathbb{H})$. A compact quantum subgroup of a coamenable locally compact quantum group $\mathbb{G}$ is a compact quantum group $\mathbb{H}$ such that there exists a surjective $*$-homomorphism

$$
\pi: \mathrm{C}_{0}(\mathbb{G}) \rightarrow \mathrm{C}(\mathbb{H}) \quad(\pi \otimes \pi) \Delta_{\mathbb{G}}=\Delta_{\mathbb{H}} \pi .
$$

(The reader is warned that there are other definitions of closed quantum subgroup [38, 39, and it is not clear whether they are all equivalent. In our situation, all the definitions coincide as they do in many other cases; see [5.) A compact quantum subgroup of $\mathbb{G}$ always gives rise to an idempotent state on $\mathbb{G}$. Indeed, when a locally compact quantum group $\mathbb{H}$ is compact, the left and right Haar weights are actually bounded functionals and coincide. By normalisation, there exists a unique state - the Haar state $-\phi_{\mathbb{H}}$ on $\mathrm{C}(\mathbb{H})$ that is both left and right invariant. Using the subgroup morphism $\pi$, we may pull back $\phi_{\mathbb{H}}$ to obtain an idempotent state $\omega=\phi_{\mathbb{H}} \pi$ on $\mathbb{G}$. Obviously $\omega$ is a state and it is an idempotent due to invariance of $\phi_{\mathbb{H}}$ :

$$
\begin{aligned}
\omega \star \omega & =\left(\left(\phi_{\mathbb{H}} \pi\right) \otimes\left(\phi_{\mathbb{H}} \pi\right)\right) \Delta_{\mathbb{G}}=\left(\phi_{\mathbb{H}} \otimes \phi_{\mathbb{H}}\right)(\pi \otimes \pi) \Delta_{\mathbb{G}} \\
& =\left(\phi_{\mathbb{H}} \otimes \phi_{\mathbb{H}}\right) \Delta_{\mathbb{H}} \pi=\phi_{\mathbb{H}}(1) \phi_{\mathbb{H}} \pi=\omega .
\end{aligned}
$$

It should be noted that in the case of compact quantum groups, the existence of Haar state follows from the other axioms as shown by Woronowicz [42, 45. Indeed, this may be done with a similar process of using Cesàro averages as mentioned after the Kawada-Itô theorem: the Cesàro averages of convolution powers of a faithful state converge to the Haar state (however, the resulting Haar state is not necessarily faithful). The assumption that there is a faithful state on the $\mathrm{C}^{*}$-algebra, which is true in the separable case, may be dropped, as shown by Van Daele [40.

Now it is possible to at least formulate the statement of the Kawada-Itô theorem: every idempotent state on a locally compact quantum group $\mathbb{G}$ is a Haar idempotent, that is, of the form $\omega=\phi_{\mathbb{H}} \pi$ where $\phi_{\mathbb{H}}$ is the Haar state of a compact quantum subgroup $\mathbb{H}$ of $\mathbb{G}$ and $\pi: \mathrm{C}_{0}(\mathbb{G}) \rightarrow \mathrm{C}(\mathbb{H})$ is the associated morphism. The problem is that this statement is false. It is, moreover, easily seen to be false. Let $G$ be an amenable locally compact group with a non-normal open subgroup 
$H$. Then $1_{H}$ is an idempotent state on $\mathrm{C}_{r}^{*}(G)$. If $1_{H}$ were a pullback of the Haar state of a compact quantum subgroup of $\mathrm{C}_{r}^{*}(G)$, then the compact quantum subgroup would necessarily be of the form $\mathrm{C}_{r}^{*}(G / H)$. But $H$ not being normal, this is not possible (see [10, Theorem 6.2] for the finite case and [31, section 7] for a related discussion on left invariant $\mathrm{C}^{*}$-subalgebras in $\left.\mathrm{C}_{r}^{*}(G)\right)$.

Although this example, obtainable with finite groups, certainly seems to be the most straightforward counterexample of the Kawada-Itô theorem for quantum groups, it was not the first one. The first counterexample is due to Pal 24 and it comes from a genuine quantum group: the Kac-Paljutkin quantum group. We shall next describe this quantum group and Pal's example as well as provide a new insight to this example.

\section{Pal's COUnterexample}

The underlying $\mathrm{C}^{*}$-algebra of the Kac-Paljutkin quantum group $\mathbb{G}$ is

$$
\mathbb{C} \oplus \mathbb{C} \oplus \mathbb{C} \oplus \mathbb{C} \oplus \mathrm{M}_{2}(\mathbb{C}),
$$

the basis of which is given by the vectors

$$
e_{k}=\delta_{1, k} \oplus \delta_{2, k} \oplus \delta_{3, k} \oplus \delta_{4, k} \oplus\left(\begin{array}{ll}
\delta_{5, k} & \delta_{8, k} \\
\delta_{7, k} & \delta_{6, k}
\end{array}\right)
$$

$k=1,2, \ldots, 8$. The comultiplication of $\mathbb{G}$ is defined by

$$
\begin{aligned}
\Delta\left(e_{1}\right)= & e_{1} \otimes e_{1}+e_{2} \otimes e_{2}+e_{3} \otimes e_{3}+e_{4} \otimes e_{4} \\
& +\frac{1}{2}\left(e_{5} \otimes e_{5}+e_{6} \otimes e_{6}+e_{7} \otimes e_{7}+e_{8} \otimes e_{8}\right) \\
\Delta\left(e_{2}\right)= & e_{1} \otimes e_{2}+e_{2} \otimes e_{1}+e_{3} \otimes e_{4}+e_{4} \otimes e_{3} \\
& +\frac{1}{2}\left(e_{5} \otimes e_{6}+e_{6} \otimes e_{5}+i e_{7} \otimes e_{8}-i e_{8} \otimes e_{7}\right) \\
\Delta\left(e_{3}\right)= & e_{1} \otimes e_{3}+e_{3} \otimes e_{1}+e_{2} \otimes e_{4}+e_{4} \otimes e_{2} \\
& +\frac{1}{2}\left(e_{5} \otimes e_{6}+e_{6} \otimes e_{5}-i e_{7} \otimes e_{8}+i e_{8} \otimes e_{7}\right) \\
\Delta\left(e_{4}\right)= & e_{1} \otimes e_{4}+e_{4} \otimes e_{1}+e_{2} \otimes e_{3}+e_{3} \otimes e_{2} \\
& +\frac{1}{2}\left(e_{5} \otimes e_{5}+e_{6} \otimes e_{6}-e_{7} \otimes e_{7}-e_{8} \otimes e_{8}\right) \\
\Delta\left(e_{5}\right)= & e_{1} \otimes e_{5}+e_{5} \otimes e_{1}+e_{2} \otimes e_{6}+e_{6} \otimes e_{2} \\
& +e_{3} \otimes e_{6}+e_{6} \otimes e_{3}+e_{4} \otimes e_{5}+e_{5} \otimes e_{4} \\
\Delta\left(e_{6}\right)= & e_{1} \otimes e_{6}+e_{6} \otimes e_{1}+e_{2} \otimes e_{5}+e_{5} \otimes e_{2} \\
& +e_{3} \otimes e_{5}+e_{5} \otimes e_{3}+e_{4} \otimes e_{6}+e_{6} \otimes e_{4} \\
\Delta\left(e_{7}\right)= & e_{1} \otimes e_{7}+e_{7} \otimes e_{1}-i e_{2} \otimes e_{8}+i e_{8} \otimes e_{2} \\
& +i e_{3} \otimes e_{8}-i e_{8} \otimes e_{3}-e_{4} \otimes e_{7}-e_{7} \otimes e_{4} \\
\Delta\left(e_{8}\right)= & e_{1} \otimes e_{8}+e_{8} \otimes e_{1}+i e_{2} \otimes e_{7}-i e_{7} \otimes e_{2} \\
& -i e_{3} \otimes e_{7}+i e_{7} \otimes e_{3}-e_{4} \otimes e_{8}-e_{8} \otimes e_{4} .
\end{aligned}
$$

Pal's idempotent state is defined by

$$
\omega\left(\sum_{k=1}^{8} \alpha_{k} e_{k}\right)=\frac{1}{4} \alpha_{1}+\frac{1}{4} \alpha_{4}+\frac{1}{2} \alpha_{6} .
$$

As we shall see in section 9] we can always associate a certain $\mathrm{C}^{*}$-subalgebra to an idempotent state. For Pal's idempotent state $\omega$, the associated $\mathrm{C}^{*}$-subalgebra (id $\left.\otimes \omega\right) \Delta(\mathrm{C}(\mathbb{G})$ ) is spanned by the elements

$$
a=e_{1}+e_{2}+e_{3}+e_{4}+e_{5}+e_{6} \text { and } b=e_{1}-e_{2}-e_{3}+e_{4}-e_{5}+e_{6} .
$$

Moreover, one can calculate that

$$
\Delta(a)=a \otimes a \quad \text { and } \quad \Delta(b)=b \otimes b .
$$


Now consider the quantum group $\widehat{\mathbb{Z}}_{2}$ given by the group $\mathrm{C}^{*}$-algebra $\mathrm{C}^{*}\left(\mathbb{Z}_{2}\right)$ (of course $\mathbb{Z}_{2} \cong \widehat{\mathbb{Z}}_{2}$ but the chosen viewpoint suits us better). Then $\mathrm{C}^{*}\left(\mathbb{Z}_{2}\right)$ is spanned by $\lambda(0)$ and $\lambda(1)$, where $\lambda$ denotes the left regular representation of $\mathbb{Z}_{2}$. Define $\pi: \mathrm{C}^{*}\left(\mathbb{Z}_{2}\right) \rightarrow \mathrm{C}(\mathbb{G})$ by $\pi(\lambda(0))=a$ and $\pi(\lambda(1))=b$. By (1), we see that $\pi$ preserves the quantum group structure of $\widehat{\mathbb{Z}}_{2}$. There is also a conditional expectation $E$ onto $\pi\left(\mathrm{C}^{*}\left(\mathbb{Z}_{2}\right)\right)$ defined by

$$
\begin{gathered}
E\left(e_{1}\right)=E\left(e_{4}\right)=\frac{1}{8}(a+b) \quad E\left(e_{2}\right)=E\left(e_{3}\right)=\frac{1}{8}(a-b) \\
E\left(e_{5}\right)=\frac{1}{4}(a-b) \quad E\left(e_{6}\right)=\frac{1}{4}(a+b) \quad E\left(e_{7}\right)=E\left(e_{8}\right)=0 .
\end{gathered}
$$

The counit $\epsilon_{\widehat{\mathbb{Z}}_{2}}$ of $\widehat{\mathbb{Z}}_{2}$ is the constant function 1 (considered as an element of $\mathrm{B}\left(\mathbb{Z}_{2}\right)$ ). Finally, note that $\omega=\epsilon_{\widehat{\mathbb{Z}}_{2}} \circ \pi^{-1} \circ E$. What this shows is that Pal's idempotent state is of the similar form as the idempotent states $1_{H}$ on group $\mathrm{C}^{*}$-algebras: $1_{H}=\epsilon_{\widehat{H}} \circ \pi^{-1} \circ E$ where $\epsilon_{\widehat{H}}$ is the counit of $\widehat{H}$ (i.e. constant 1 on $H), \pi: \mathrm{C}_{r}^{*}(H) \rightarrow \mathrm{C}_{r}^{*}(G)$ is the natural embedding (i.e. zero extension), and $E: \mathrm{C}_{r}^{*}(G) \rightarrow \pi\left(\mathrm{C}_{r}^{*}(H)\right)$ is the natural conditional expectation (i.e. restriction to $\left.H\right)$. So although Pal's idempotent is not like the idempotent states in the commutative case (i.e. not a Haar idempotent), it is similar to the idempotent states in the co-commutative case. Thus it is associated with a subgroup but in a different way.

These examples of idempotent states that are not Haar idempotents show that a new approach is needed for general locally compact quantum groups. In section 8 we consider another notion, that of left invariant $\mathrm{C}^{*}$-subalgebras, that is closely tied with idempotent states as we shall see. There is also the approach of Franz and Skalski, who show in [10, that every idempotent state on a finite quantum group arises from the Haar state of a so-called quantum subhypergroup. On a related note, Franz and Skalski [9] also show that idempotent states on a finite quantum group correspond to quantum pre-subgroups in the sense of [1]. In all these approaches one associates idempotent states with structures more general than subgroups, and that is what we shall do with left invariant $\mathrm{C}^{*}$-subalgebras in section 9 .

\section{Positive EXAmples From Deformation QUANTUM GROUPS}

In this section we shall consider idempotent states on some important examples of compact quantum groups, in particular on the quantum deformation of $\mathrm{SU}(2)$ introduced by Woronowicz [43, 42]. It turns out that on these deformations of classical groups, $\mathrm{SU}_{q}(2), \mathrm{U}_{q}(2)$ and $\mathrm{SO}_{q}(3)$, all idempotent states are Haar idempotents. The results in this section are due to Franz, Skalski and Tomatsu 11 .

Define $\mathrm{C}\left(\mathrm{SU}_{q}(2)\right)$ as the universal unital $\mathrm{C}^{*}$-algebra generated by elements $a$ and $c$ such that

$$
\left(\begin{array}{cc}
a & -q c^{*} \\
c & a^{*}
\end{array}\right)
$$

is formally a unitary matrix (a $2 \times 2$ matrix with entries in $\mathrm{C}\left(\mathrm{SU}_{q}(2)\right)$ ). The comultiplication of $\mathrm{SU}_{q}(2)$ is determined by the identity

$$
\Delta\left(\begin{array}{cc}
a & -q c^{*} \\
c & a^{*}
\end{array}\right)=\left(\begin{array}{cc}
a & -q c^{*} \\
c & a^{*}
\end{array}\right) \otimes\left(\begin{array}{cc}
a & -q c^{*} \\
c & a^{*}
\end{array}\right) .
$$

This identity is to be read as follows: on the left-hand side we apply $\Delta$ to each entry and on the right-hand side we take a formal matrix multiplication where we use the tensor product when 'multiplying' entries; then we just equate the entries of the two $2 \times 2$ matrices. Although the relations given above fully determine the structure of $\mathrm{SU}_{q}(2)$, to prove that we actually get a compact quantum group takes some work. Recall however that the existence of the Haar state follows from the general theory of compact quantum groups.

Using the representation theory of $\mathrm{SU}_{q}(2)$, Franz, Skalski and Tomatsu [1] calculated all the idempotent states on $\mathrm{SU}_{q}(2)$ for $q \in(-1,0) \cup(0,1)$. It turns out that these are all Haar idempotents. Namely, the idempotent states on $\mathrm{SU}_{q}(2)$ are the Haar state and the Haar idempotents coming from the subgroups $\mathbb{T}$ and $\mathbb{Z}_{n}, 1 \leq n<\infty$. We see that $\mathbb{T}$ is a subgroup of $\mathrm{SU}_{q}(2)$ by mapping the generator $a$ to the generator $z$ of $\mathrm{C}(\mathbb{T})$ and $c$ to 0 . Moreover, $\mathbb{Z}_{n}$ 's are subgroups of 
$\mathbb{T}$. Already Podleś 27 , showed that these are all the closed quantum subgroups of $\mathrm{SU}_{q}(2)$. This result follows also from [11, but of course it takes more work to show that all idempotent states actually arise from these subgroups. Franz, Skalski and Tomatsu also give the complete list of idempotent states for the related deformation quantum groups $\mathrm{U}_{q}(2)$ and $\mathrm{SO}_{q}(3)$. Also in these cases all idempotent states are Haar idempotents.

\section{LeFT INVARIANT $\mathrm{C}^{*}$-SUBALGEBRAS}

In this section we shall consider another notion related to idempotent states besides subgroups. The notion is that of left invariant $\mathrm{C}^{*}$-subalgebra (here we could use alternative terminology and call these coideals or homogeneous spaces).

Let $\mathbb{G}$ be a coamenable locally compact quantum group. For $\omega \in \mathrm{C}_{0}(\mathbb{G})^{*}$, define the left and right convolution operators on $\mathrm{C}_{0}(\mathbb{G})$ by

$$
\begin{aligned}
& L_{\omega}(a)=(\omega \otimes \mathrm{id}) \Delta(a) \\
& R_{\omega}(a)=(\mathrm{id} \otimes \omega) \Delta(a)
\end{aligned} \quad\left(a \in \mathrm{C}_{0}(\mathbb{G})\right) .
$$

A C*-subalgebra $X \subseteq \mathrm{C}_{0}(\mathbb{G})$ is said to be left invariant if $L_{\omega}(X) \subseteq X$ for all $\omega \in \mathrm{C}_{0}(\mathbb{G})^{*}$. A nondegenerate $\mathrm{C}^{*}$-subalgebra $X$ of $\mathrm{C}_{0}(\mathbb{G})$ is left invariant if and only if $\Delta: X \rightarrow \mathrm{M}\left(\mathrm{C}_{0}(\mathbb{G}) \otimes X\right)$. $\left(\mathrm{A} \mathrm{C} \mathrm{C}^{*}\right.$-subalgebra is nondegenerate if it contains a bounded approximate identity for the ambient $\mathrm{C}^{*}$-algebra.)

Consider the commutative case when $G$ is a locally compact group. Then a $\mathrm{C}^{*}$-subalgebra $X$ of $\mathrm{C}_{0}(G)$ is left invariant if and only if it is left translation invariant; that is, the function $L_{s} f(t)=f(s t)$ is in $X$ whenever $f \in X$ and $s \in G$. Lau and Losert [22] have characterised left invariant $\mathrm{C}^{*}$-subalgebras of $\mathrm{C}_{0}(G)$ : a $\mathrm{C}^{*}$-subalgebra $X \subseteq \mathrm{C}_{0}(G)$ is left invariant if and only if there is a compact subgroup $H$ of $G$ such that $X$ consists of all the functions in $\mathrm{C}_{0}(G)$ that are constant on right cosets of $H$. The latter statement means that $X$ is $*$-isomorphic to $\mathrm{C}_{0}(G / H)$. Earlier, de Leeuw and Mirkil [ 6 gave this characterisation in the case of locally compact abelian groups. Moreover, Takesaki and Tatsuuma 35 produced several related results, characterising closed (left) invariant self-adjoint subalgebras of $\mathrm{L}^{\infty}(G)$, the Fourier algebra $\mathrm{A}(G)$, the group von Neumann algebra $\operatorname{VN}(G)$ and the $\mathrm{L}^{1}$ group algebra (here the meaning of 'closed' depends on the context: with $\mathrm{A}(G)$ and $\mathrm{L}^{1}(G)$ it means norm-closed and with $\mathrm{L}^{\infty}(G)$ and $\mathrm{VN}(G)$ it means weak*closed). The dual version of the Lau-Losert characterisation for a locally compact amenable group $G$ is given in 31: a $\mathrm{C}^{*}$-subalgebra $X \subseteq \mathrm{C}_{r}^{*}(G)$ is invariant if and only if $X \cong \mathrm{C}_{r}^{*}(H)$ for some open subgroup $H$. One can also consider strictly closed left invariant $\mathrm{C}^{*}$-subalgebras of $\mathrm{C}_{b}(G)$ and $\mathrm{M}\left(\mathrm{C}_{r}^{*}(G)\right)$ and obtain in both cases a one-to-one correspondence with closed subgroups of $G$ 32 .

Finally, we also have the following result from [31, concerning left invariant $\mathrm{C}^{*}$-subalgebras of coamenable locally compact quantum groups. Recall that a conditional expectation on a $\mathrm{C}^{*}$ algebra $A$ is a norm 1 projection from $A$ onto a $\mathrm{C}^{*}$-subalgebra of $A$. The following result also employs a symmetry condition that is related to the problem brought out by Pal's counterexample. We postpone the formulation of this symmetry condition until after the theorem.

Theorem 3. There is a one-to-one correspondence between compact quantum subgroups of $\mathbb{G}$ and symmetric, left invariant $C^{*}$-subalgebras $X$ of $\mathrm{C}_{0}(\mathbb{G})$ with a conditional expectation $P$ from $\mathrm{C}_{0}(\mathbb{G})$ onto $X$ such that $($ id $\otimes P) \Delta=\Delta P$.

Let $G$ be an amenable locally compact group and $H$ an open subgroup of $G$. As noted above, $\mathrm{C}_{r}^{*}(H)$ is an invariant $\mathrm{C}^{*}$-subalgebra of $\mathrm{C}_{r}^{*}(G)$. However, $\mathrm{C}_{r}^{*}(H)$ is not associated with a compact quantum subgroup unless $H$ is normal, in which case $\mathrm{C}_{r}^{*}(H)$ is associated with $\mathrm{C}_{r}^{*}(G / H)$. We shall need an analogue of this normality condition for more general quantum groups. This can be done through the so-called multiplicative unitary of a locally compact quantum group $\mathbb{G}$. There is a canonical way to define a unitary operator $W$ on $\mathrm{L}^{2}(\mathbb{G}) \otimes \mathrm{L}^{2}(\mathbb{G})$ such that $W$ determines the quantum group $\mathbb{G}$ 21, Proposition 3.17]. Here $L^{2}(\mathbb{G})$ denotes the Hilbert space obtained by applying the GNS-construction to the left Haar weight of $\mathbb{G}$. The $\mathrm{C}^{*}$-algebra $\mathrm{C}_{0}(\mathbb{G})$ is faithfully represented on $\mathrm{L}^{2}(\mathbb{G})$ and it is natural to identify $\mathrm{C}_{0}(\mathbb{G})$ with its image in $\mathrm{B}\left(\mathrm{L}^{2}(\mathbb{G})\right)$. The 
multiplicative unitary $W$ determines the comultiplication via

$$
\Delta(a)=W^{*}(1 \otimes a) W \quad\left(a \in \mathrm{C}_{0}(\mathbb{G})\right) .
$$

The notion of multiplicative unitary is central in the theory of locally compact quantum groups; seminal work in this area is due to Baaj and Skandalis 2 and Woronowicz 44.

We say that a $\mathrm{C}^{*}$-subalgebra $X$ of $\mathrm{C}_{0}(\mathbb{G})$ is symmetric if

$$
W(x \otimes 1) W^{*} \in \mathrm{M}\left(X \otimes B_{0}\left(\mathrm{~L}^{2}(\mathbb{G})\right)\right)
$$

whenever $x \in X$ (here $\mathrm{B}_{0}$ denotes the compact operators). Tomatsu [37] introduced this type of condition, calling it coaction symmetry (due to the fact that $X$ is symmetric if and only if it is closed under the natural action of the dual quantum group of $\mathbb{G}$ ). Returning to the cocommutative case, the left invariant $\mathrm{C}^{*}$-subalgebra $\mathrm{C}_{r}^{*}(H)$ associated with an open subgroup $H$ of $G$ is symmetric if and only if $H$ is normal 31 .

\section{IDEMPOTENT STATES AND LEFT INVARIANT C*-SUBALGEBRAS}

Although Pal's counterexample showed that we cannot associate all idempotent states to compact quantum subgroups, we may still have a chance of associating idempotent states to suitable left invariant $\mathrm{C}^{*}$-subalgebras. The results in this section are from [33], many of them generalisations from [9] or [1].

Let $\mathbb{G}$ be a coamenable locally compact quantum group. If $\omega$ is an idempotent state on $\mathbb{G}$, then $R_{\omega}\left(\mathrm{C}_{0}(\mathbb{G})\right)$ is a left invariant $\mathrm{C}^{*}$-subalgebra of $\mathrm{C}_{0}(\mathbb{G})$ and $R_{\omega}$ is a conditional expectation onto this $\mathrm{C}^{*}$-subalgebra. The following result generalises an earlier result due to Franz and Skalski 9 ] concerning compact quantum groups.

Theorem 4. Suppose that $\mathbb{G}$ is unimodular $($ i.e. $\phi=\psi)$. There is a one-to-one correspondence between idempotent states $\omega$ on $\mathbb{G}$ and left invariant $C^{*}$-subalgebras $X$ of $\mathrm{C}_{0}(\mathbb{G})$ with a conditional expectation $P$ from $\mathrm{C}_{0}(\mathbb{G})$ onto $X$ such that $\phi \circ P=\phi$. The correspondence is given by

$$
X_{\omega}=R_{\omega}\left(\mathrm{C}_{0}(\mathbb{G})\right), \quad \omega_{X}=\epsilon P_{X} .
$$

where $\epsilon$ is the counit of $\mathbb{G}$.

The preceding result leaves room for improvement: one would like to remove the unimodularity assumption in which case the conditional expectation should preserve both left and right Haar weights.

The following result characterises those idempotent states that arise from compact quantum subgroups. It also brings together the symmetry condition from the preceding section. The equivalence between the first and the third condition is proved for compact quantum groups in [1].

Theorem 5. Let $\omega$ be an idempotent state on $\mathbb{G}$ and let $X_{\omega}=R_{\omega}\left(\mathrm{C}_{0}(\mathbb{G})\right)$. The following are equivalent:

(1) $\omega$ is a Haar idempotent;

(2) $X_{\omega}$ is symmetric;

(3) $N_{\omega}:=\left\{a \in \mathrm{C}_{0}(\mathbb{G}) ; \omega\left(a^{*} a\right)=0\right\}$ is an ideal.

The set $N_{\omega}$ in the third condition of the the preceding result is always a left ideal, so the condition is automatically satisfied if $\mathrm{C}_{0}(\mathbb{G})$ is commutative. Consequently, we get the KawadaItô theorem from section 2 as a corollary.

Corollary 6 (Kawada-Itô). If $\mathrm{C}_{0}(\mathbb{G})$ is commutative, then all idempotent states on $\mathbb{G}$ are Haar idempotents.

Finally, we have the following correspondence result, which does not assume unimodularity, but works only for Haar idempotents.

Theorem 7. There is a one-to-one correspondence between Haar idempotents $\omega$ on $\mathbb{G}$ and symmetric, left invariant $C^{*}$-subalgebras $X$ of $\mathrm{C}_{0}(\mathbb{G})$ with a conditional expectation $P$ from $\mathrm{C}_{0}(\mathbb{G})$ onto $X$ such that $\phi \circ P=\phi$ and $\psi \circ P=\psi$. 
Note that the preceding theorem improves Theorem 3 in the sense that the condition that $($ id $\otimes P) \Delta=\Delta P$ may be replaced by the more natural condition that $P$ preserves both left and right Haar weights.

Acknowledgement. This paper is based on a talk given in the conference 'Operator Theory and its Applications' held in honour of Victor Shulman in Gothenburg 2011; I thank the organisers Ivan Todorov and Lyudmila Turowska for a great conference. I thank Nico Spronk for generous support throughout my postdoctoral stay at University of Waterloo and in particular for enabling my conference visit. I thank Emil Aaltonen Foundation for support during the preparation of this paper. I thank Adam Skalski and the referee for helpful comments improving the paper.

\section{REFERENCES}

[1] S. Baaj, E. Blanchard and G. Skandalis, Unitaires multiplicatifs en dimension finie et leurs sous-objets, Ann. Inst. Fourier (Grenoble) 49 (1999), 1305-1344.

[2] S. Baaj and G. Skandalis, Unitaires multiplicatifs et dualité pour les produits croisés de $C^{*}$-algèbres, Ann. Sci. École Norm. Sup. (4) 26 (1993), 425-488.

[3] T. Banica, U. Franz and A. Skalski, Idempotent states and the inner linearity property, preprint, arXiv: 1112.5018

[4] P. J. Cohen, On a conjecture of Littlewood and idempotent measures, Amer. J. Math. 82 (1960), $191-212$.

[5] M. Daws, P. Kasprzak, A. Skalski and P. M. Soltan, Closed quantum subgroups of locally compact quantum groups, preprint.

[6] K. de Leeuw and H. Mirkil, Translation-invariant function algebras on abelian groups, Bull. Soc. Math. France 88 (1960), 345-370.

[7] M. Enock and J.-M. Schwartz, Kac algebras and duality of locally compact groups, Springer-Verlag, Berlin, 1992.

[8] U. Franz and A. Skalski, On ergodic properties of convolution operators associated with compact quantum groups, Colloq. Math. 113 (2008), 13-23.

[9] U. Franz and A. Skalski, A new characterisation of idempotent states on finite and compact quantum groups, C. R. Math. Acad. Sci. Paris 347 (2009), 991-996.

[10] U. Franz and A. Skalski, On idempotent states on quantum groups, J. Algebra 322 (2009), 1774-1802.

[11] U. Franz, A. Skalski and R. Tomatsu, Idempotent states on compact quantum groups and their classification on $U_{q}(2), S U_{q}(2)$, and $S O_{q}(3)$, to appear in J. Noncomm. Geom., arXiv:0903.2363.

[12] U. Grenander, Probabilities on algebraic structures, Almqvist \& Wiksell, Stockholm, 1968.

[13] G. Högnäs and A. Mukherjea, Probability measures on semigroups: convolution products, random walks, and random matrices, second ed., Springer, New York, 2011.

[14] B. Host, Le théorème des idempotents dans $B(G)$, Bull. Soc. Math. France 114 (1986), 215-223.

[15] M. Ilie and N. Spronk, Completely bounded homomorphisms of the Fourier algebras, J. Funct. Anal. 225 (2005), 480-499.

[16] M. Kalantar, M. Neufang and Z.-J. Ruan, Poisson boundaries over locally compact quantum groups, preprint, arXiv: 1111.5828

[17] Y. Kawada and K. Itô, On the probability distribution on a compact group. I, Proc. Phys.-Math. Soc. Japan (3) 22 (1940), 977-998.

[18] J. L. Kelley, Averaging operators on $C_{\infty}(X)$, Illinois J. Math. 2 (1958), 214-223.

[19] J. Kustermans and L. Tuset, A survey of $C^{*}$-algebraic quantum groups, part I, Irish Math. Soc. Bull. (1999), no. $43,8-63$.

[20] J. Kustermans and L. Tuset, A survey of $C^{*}$-algebraic quantum groups, part II, Irish Math. Soc. Bull. (2000), no. $44,6-54$.

[21] J. Kustermans and S. Vaes, Locally compact quantum groups, Ann. Sci. École Norm. Sup. (4) 33 (2000), $837-934$

[22] A. T.-M. Lau and V. Losert, Complementation of certain subspaces of $L_{\infty}(G)$ of a locally compact group, Pacific J. Math. 141 (1990), 295-310.

[23] R. M. Loynes, Fourier transforms and probability theory on a noncommutative locally compact topological group, Ark. Mat. 5 (1963), 37-42.

[24] A. Pal, A counterexample on idempotent states on a compact quantum group, Lett. Math. Phys. 37 (1996), $75-77$.

[25] K. R. Parthasarathy, A note on idempotent measures in topological groups, J. London Math. Soc. 42 (1967), $534-536$.

[26] V. Paulsen, Completely bounded maps and operator algebras, Cambridge University Press, Cambridge, 2002.

[27] P. Podleś, Symmetries of quantum spaces. Subgroups and quotient spaces of quantum $\mathrm{SU}(2)$ and $\mathrm{SO}(3)$ groups, Comm. Math. Phys. 170 (1995), 1-20.

[28] J. S. Pym, Idempotent measures on semigroups, Pacific J. Math. 12 (1962), 685-698.

[29] W. Rudin, Idempotent measures on Abelian groups, Pacific J. Math. 9 (1959), 195-209. 
[30] W. Rudin, Measure algebras on abelian groups, Bull. Amer. Math. Soc. 65 (1959), 227-247.

[31] P. Salmi, Compact subgroups and left invariant $C^{*}$-subalgebras of locally compact quantum groups, J. Funct. Anal. 261 (2011), 1-24.

[32] P. Salmi, Subgroups and strictly closed invariant $C^{*}$-subalgebras, preprint, arXiv:1110.5459

[33] P. Salmi and A. Skalski, Idempotent states on locally compact quantum groups, to appear in Q. J. Math., arXiv:1102.2051

[34] M. Takesaki, Theory of operator algebras. II, Springer-Verlag, Berlin, 2003.

[35] M. Takesaki and N. Tatsuuma, Duality and subgroups, Ann. of Math. (2) 93 (1971), 344-364.

[36] T. Timmermann, An invitation to quantum groups and duality. From Hopf algebras to multiplicative unitaries and beyond, European Mathematical Society (EMS), Zürich, 2008.

[37] R. Tomatsu, A characterization of right coideals of quotient type and its application to classification of Poisson boundaries, Comm. Math. Phys. 275 (2007), 271-296.

[38] S. Vaes, A new approach to induction and imprimitivity results, J. Funct. Anal. 229 (2005), 317-374.

[39] S. Vaes and L. Vainerman, On low-dimensional locally compact quantum groups, Locally compact quantum groups and groupoids (Strasbourg, 2002), IRMA Lect. Math. Theor. Phys., vol. 2, de Gruyter, Berlin, 2003, pp. $127-187$.

[40] A. Van Daele, The Haar measure on a compact quantum group, Proc. Amer. Math. Soc. 123 (1995), 3125-3128.

[41] J. G. Wendel, Haar measure and the semigroup of measures on a compact group, Proc. Amer. Math. Soc. 5 (1954), 923-929.

[42] S. L. Woronowicz, Compact matrix pseudogroups, Comm. Math. Phys. 111 (1987), 613-665.

[43] S. L. Woronowicz, Twisted SU(2) group. An example of a noncommutative differential calculus, Publ. Res. Inst. Math. Sci. 23 (1987), 117-181.

[44] S. L. Woronowicz, From multiplicative unitaries to quantum groups, Internat. J. Math. 7 (1996), 127-149.

[45] S. L. Woronowicz, Compact quantum groups, Symétries quantiques (Les Houches, 1995), North-Holland, Amsterdam, 1998, pp. 845-884.

Department of Mathematical Sciences, University of Oulu, PL 3000, FI-90014 Oulun yliopisto, FinLAND

E-mail address: pekka.salmi@iki.fi 